\title{
Comparative study about electric vehicle charge, individualized or station based
}

\author{
E. Ramos Iglesias, M. Pérez Donsión \\ ${ }^{1}$ Department of Electrical Engineering \\ E.T.S.I.I., Vigo University \\ Campus of Lagoas - Marcosende, 36310 Vigo (Spain) \\ Phone/Fax number:+0034 986 812685, e-mail: enrique.ramosig@ gmail.com, donsión@uvigo.es
}

\begin{abstract}
This study starts from the increasing development of the Electric Vehicle and and the needs produced on the Electrical System, such as the increase in generation of energy, increase of the energy carried and the increase of the power of the installations, especially those with low voltage.

Nowadays, all the initiative of the electric vehicle is being developed oriented to each owner being able to charge his vehicle in his own garage at home or in public or community car parks. Another possible solution is using Charge Stations. Similar to Petrol Stations, where the user could change the batteries or charge his own. In this study there will be a comparison between individualized charge as opposed to the charge in stations in a small grid.
\end{abstract}

\section{Key words}

Electric vehicle, battery charge, charge stations, individualized charge.

\section{Introduction}

The Electric Vehicle will make the power demanded on the electric system be much bigger, in a way that is necessary to turn to studies that try to predict what will happen with the current grid when the Electric Technology or Hybrid-Plug-In Technology are fully applied in society. This is the reason why in this project we will perform a simulation of a tip-type system which starts from a substation. The system starts in the Output of a Transformer with a $66 \mathrm{kV}$ line. At the end of this line, two Centres of Transformation (CT) will be connected to supply two groups of housing estates. The $66 \mathrm{kV}$ line which supplies the two Centres of Transformations (CT) has a length of $20 \mathrm{~km}$. Each Centre of Transformation (CT) supplies 50 houses located within a radius of $100 \mathrm{~m}$ around the Transformer. It has been considered that in the whole 100 houses, 50 of them have only one electric vehicle whereas in the other 50 houses there are 2 electric vehicles. The first Centre of Transformation (CT) is located $15 \mathrm{~km}$ far from the substation and the second one will be connected $10 \mathrm{~km}$ far from the substation.

The results will be analysed using the charge of the vehicles in the houses, being equal to the charge in private or community car parks (figure 1) where the charges are equivalent to 10 houses and 15 electric vehicle chargers.
Subsequently, a Charge Station will be placed in the $66 \mathrm{kV}$ line, and a simulation of the charge of the same number of vehicles will be performed (figure 2).

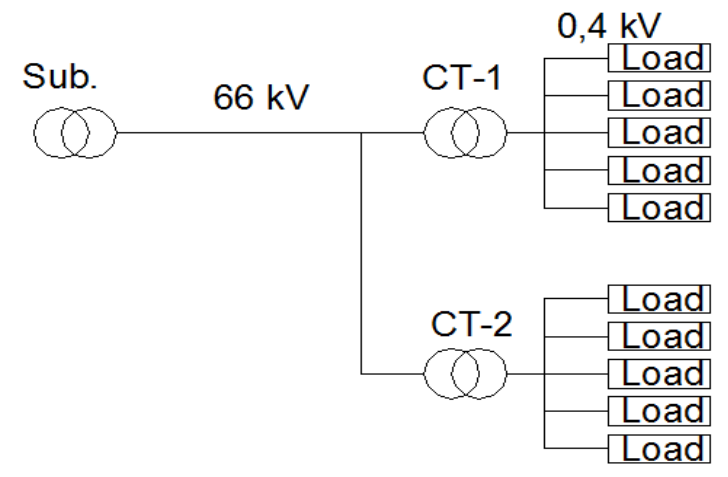

Fig.1: Sistema eléctrico carga individualizada

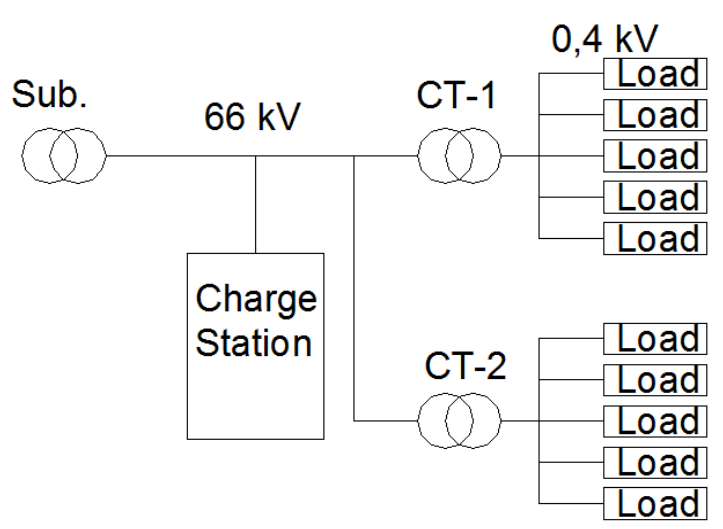

Fig.2: Sistema eléctrico carga concentrada

Where the charges are only the houses and linear consumptions. In this situation, the 150 chargers that were distributed along the low-voltage line are in the station. The operation of the station can be in two different ways: The first one as a quick charge of vehicles and the second one as an exchange station, where there will be a stock of already charged batteries available, and the batteries exchanged from the vehicles can be charged in a slower manner. This last method of operation of the station could allow us to use the excess of renewable 
production or save the consumption valleys. It would also allow us to unload the system at peak times, suppressing the charge during peak consumptions.

Moreover, It will be taken into consideration as a starting point assumption, that all the chargers in the houses have a three-phase connection, given that the single-phase rectifiers offer a performance in ideal conditions of $81 \%$ as opposed to the $96 \%$ of the three-phase-six-pulse rectifier and the $99 \%$ of a three-phase-twelve-pulse rectifier. As a result of the previously mentioned, it is clear that in order to maximize the electric system as a whole, the single-phase chargers must be dismissed.

The system will be simulated with the charging streams used nowadays:

-Slow charge: $16 \mathrm{~A}$, approximately 8 hours

-Semi-fast charge: $32 \mathrm{~A}$, approximately 4 hours

-Fast charge: 200 A, between 15 and 30 minutes.

\section{SIMULATION MODELS}

For this simulation, we have used models of the elements involved in the simplified electric system we used as a starting point. In the case of the substation, the secondary part of the transformer has been modeled as a Thevenin equivalent. Besides, an infinite short-circuit power has been considered, assuming the strongest possible upstream grid against perturbations. The decision has been made in order to avoid that in the event of an increase of the power demanded, the different charge currents reduce the voltage in the substation, causing the results to be unreliable.

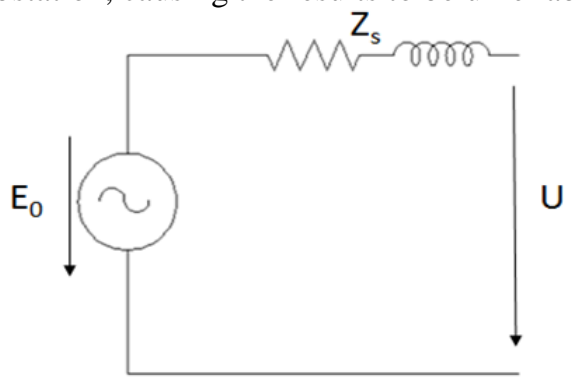

Fig.3: Thevenin equivalent

In case of the electric lines, we have used the model in $\pi$ (figure 4) with concentrated parameters, where we consider own and mutual inductances and the produced capacities [3]:

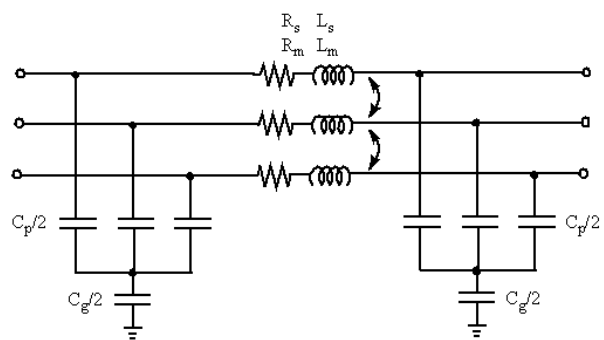

Fig.4: Concentrated parameters in Model $\pi$.
For the transformers, we use the following equivalent circuit by phase (figure 5)

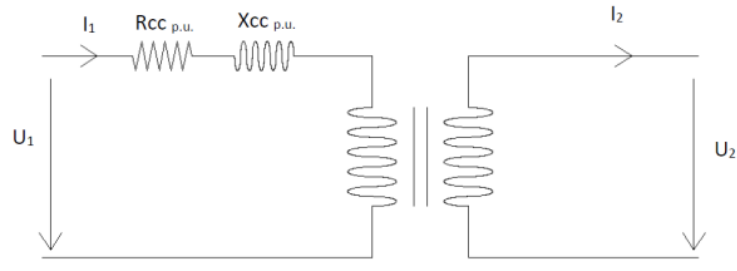

Fig.5: Equivalent circuit by phase

The low-voltage part, where the houses are connected, consists of two transformation centres used in the sameway:

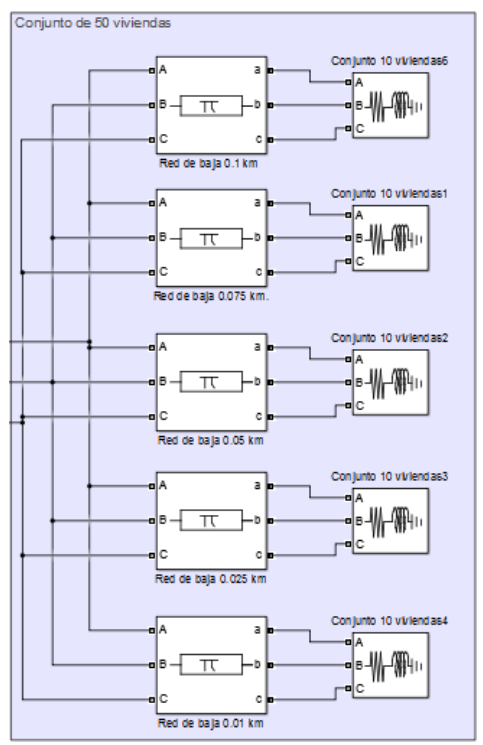

Fig.6: Charges to simulate houses

For the individualized charge, we use a three-phase-sixpulse charger (figure 7) [1]:

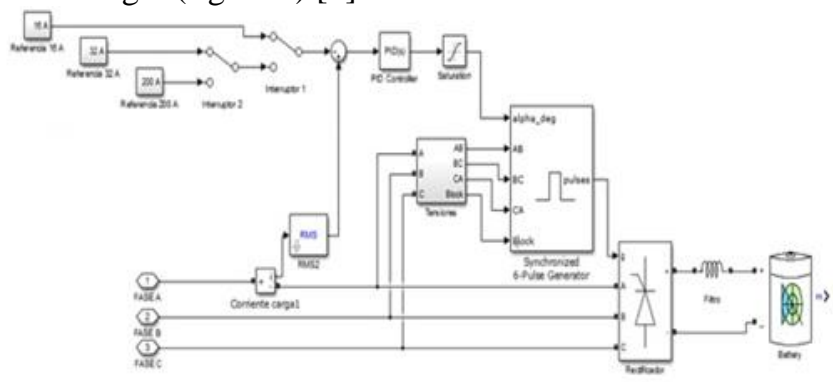

Fig.7: three-phase-six-pulse charger

The three-phase-twelve-pulse rectifier needs two transformers, one of them with the secondary Deltaconnected and the other one Star-connected. This is necessary to obtain the gap needed and allow the use of six phases [1]:

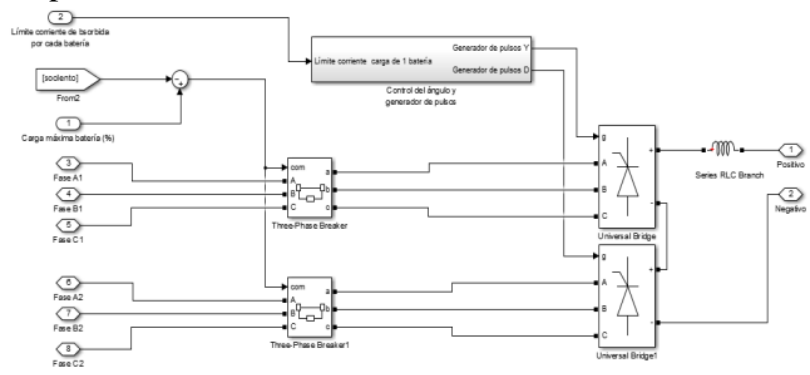

Fig.8: Three-phase-twelve-pulse charger 
In all the situations, the chargers have been designed to be able to preset the target charge percentage (default 100\%) as well as being able to select the charge current desired among 16, 32 or 200 A. Moreover, the charging system will be disconnected when the battery reaches the target charge percentage. Lastly, the houses are modeled as balanced RL charges.

In order to obtain all the results, we have used voltmeters and ammeters, besides; we have calculated the Fast Fourier Transform and the harmonic distortion rate in the current wave to see the weight of the harmonics produced in the grid.

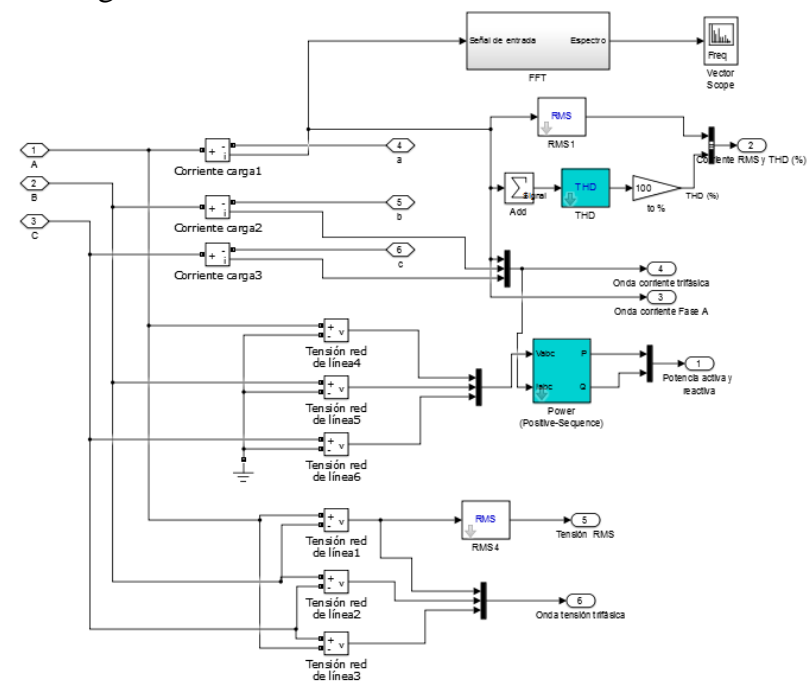

Fig.9: Electric variable meter

\section{Loss calculation}

For the individualized charge, we take measurements in the substation, in the low-voltage panel of each transformation centres and each group of houses. According to this, the following formulas are obtained from the power losses produced on the grid:

- Losses in Low-voltage lines:

$$
\begin{aligned}
& P_{p-B T}=P_{C T 1}+P_{C T 2}-P_{v i v}-P_{c a r g} \\
& Q_{p-B T}=Q_{C T 1}+Q_{C T 2}-Q_{v i v}-Q_{c a r g} \\
& S_{p-B T}=\left(P_{p-B T}+Q_{p-B T}{ }^{2}\right)^{1 / 2}
\end{aligned}
$$

- Losses in High-voltage lines:

$$
\begin{aligned}
& P_{p-A T}=P_{S U B}-P_{C T 1}-P_{C T 2} \\
& Q_{p-A T}=Q_{S U B}-Q_{C T 1}-Q_{C T 2} \\
& S_{p-B T}=\left(P_{p-A T}{ }^{2}+Q_{p-A T}\right)^{1 / 2}
\end{aligned}
$$

The losses in the High-voltage lines include the losses produced in the transformers.

- Total losses:

$$
\begin{aligned}
& P_{p}=P_{p-B T}+P_{p-A T} \\
& Q_{p}=Q_{p-B T}+Q_{p-A T} \\
& S_{p}=\left(P_{p}^{2}+Q_{p}^{2}\right)^{1 / 2}
\end{aligned}
$$

In the case of a concentrated charge, we will use threephase-six-pulse chargers for the simulation, in order to see the effect that the group of charges has on the grid. Subsequently, the charger will be exchanged for a twelvepulse charger because due to the high power needed in the station; we project the installation of two transformers. The measurements will be taken in the substation, the charge station, the transformation centres and the houses.
- Losses in the low-voltage lines:

$$
\begin{aligned}
& P_{p-B T}=P_{C T 1}+P_{C T 2}-P_{v i v} \\
& Q_{p-B T}=Q_{C T 1}+Q_{C T 2}-Q_{v i v} \\
& S_{p-B T}=\left(P_{p-B T}{ }^{2}+Q_{p-B T}\right)^{1 / 2}
\end{aligned}
$$

- Losses in the High-voltage lines:

$$
\begin{aligned}
& P_{p-A T}=P_{S U B}-P_{E S T}-P_{C T 1}-P_{C T 2} \\
& Q_{p-A T}=Q_{S U B}-Q_{E S T}-Q_{C T 1}-Q_{C T 2} \\
& S_{p-\mathrm{AT}}=\left(P_{p-A T}{ }^{2}+Q_{p-A T}\right)^{1 / 2}
\end{aligned}
$$

The losses in the High-voltage lines include the losses produced in the transformers.

- Total losses:

$$
\begin{gathered}
P_{p}=P_{p-B T}+P_{p-A T} \\
Q_{p}=Q_{p-B T}+Q_{p-A T} \\
S_{p}=\left(P_{p}^{2}+Q_{p}^{2}\right)^{1 / 2}
\end{gathered}
$$

\section{Results}

In this paragraph we will analyze the losses produced in all the systems, considering the three different types charges and currents. In order to achieve that, the data and the simulations have been grouped and represented in a bar chart which clearly shows a growth in the losses, when the energy consumption is increased.

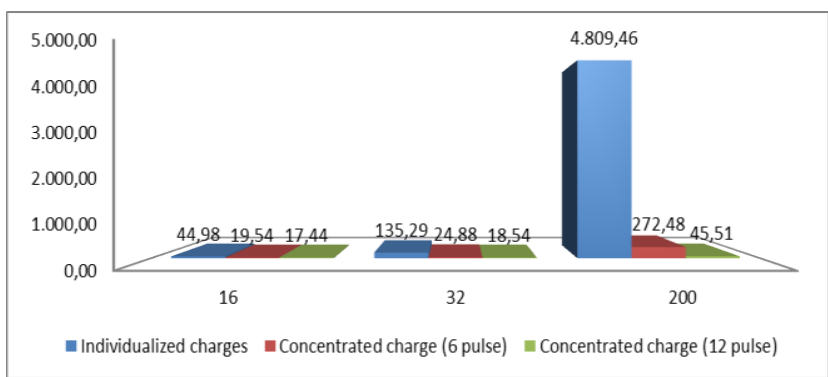

Fig.10: Apparent power from losses

We can discern that the results are very disadvantageous for the individualized charges. The losses produced are much higher than in the other types of charge using the same charge current. In this chart we show the percentage of losses against the losses produced in the individualized charge.

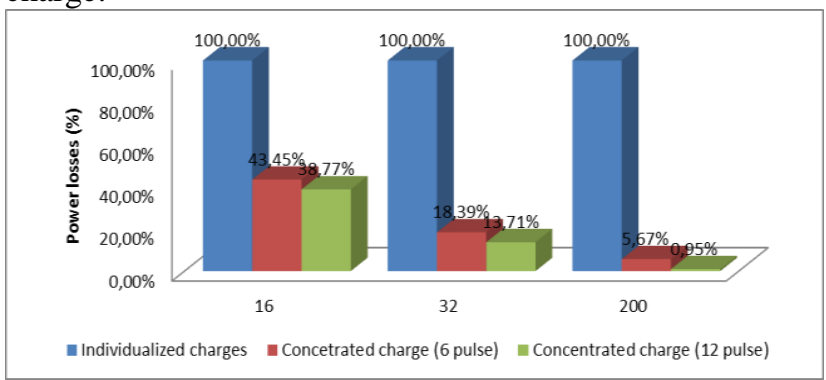

Fig.11: Power of losses against individualized charge.

Regarding the harmonic distortion rate, figure 12 shows that the twelve-pulse concentrated charge presents the lowest harmonic distortion from the three types of charges. In the case of the six-pulse concentrated charge, it is worth stressing that this one is higher even being the same type of rectifier. This is due to the fact that it is connected to the substation in a closer location, electrically speaking. The reason for this is that the reactive inductances are multiplied by the harmonic order; as a result, they have bigger impedance for high 
frequencies. This effect limits the harmonic current. Provided that the individualized charge has more electric lines, the harmonic effect is reduced in the substation.

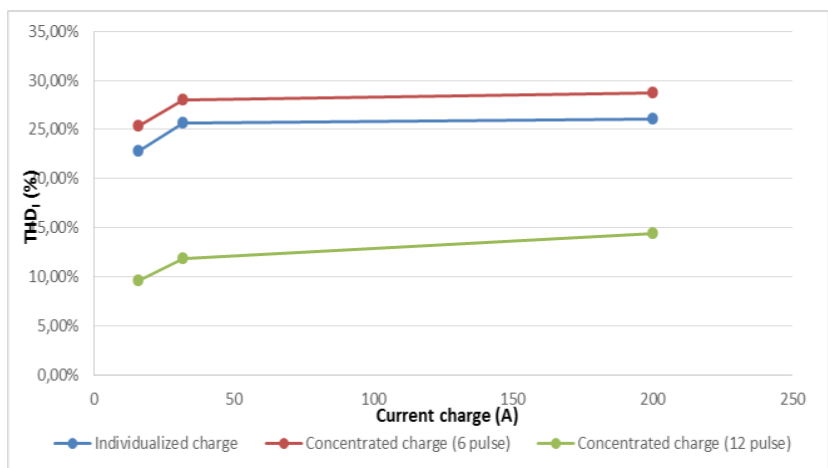

Fig.12: Current harmonic distortion rate

In the next figure we calculate the power needed to power each vehicle, starting from the results obtained in the simulations, this can provide us with an approximate idea of the power needed as the number of vehicles connected the grid is increased. We can observe again that the station with the twelve-pulse charger is the best option regarding demanded power, being one third of the power demanded in the other types of charge.

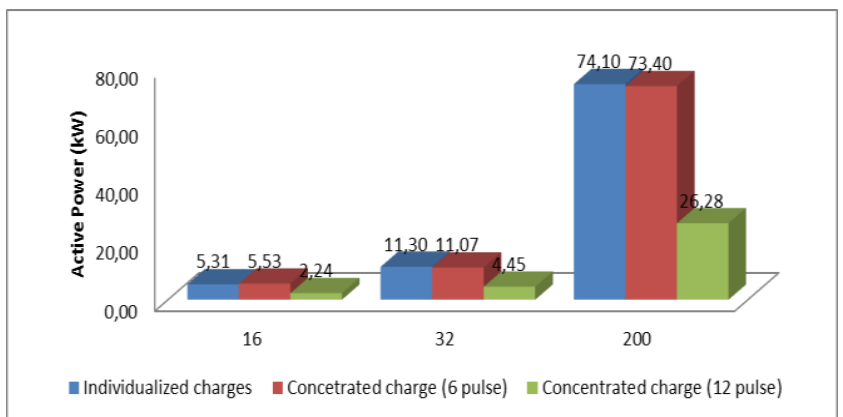

Fig.13: Active power consumed by vehicle

As we can assess, the demanded power by vehicle is virtually the same if we use a three-phase-six-pulse charger, individually used or in a concentrated manner. The significant save is obtained by using a twelve-pulse charger.

Lastly, the global efficiency of the electric system has ben analysed (figure 14):

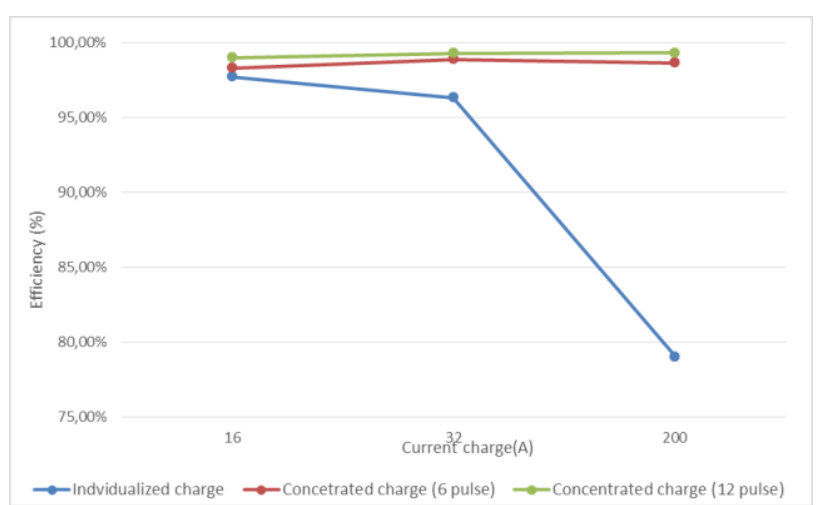

Fig.14: Global efficiency of the electric system

Where we can observe the real effect of grouping the charge in stations. It is worth stressing that efficiency of the grid is important for the whole population.

\section{Conclusion}

We have performed the simulations starting from the base that all the houses have a three-phase installation and that all of them could be able to supply the maximum current of $200 \mathrm{~A}$. This is something which is far from the truth. Individualized charge would lead us to the extra cost of modifying all the private facilities and the low-voltage distribution grids. It seems a not viable option for a constantly growing car pool. As the electricvehicle-based car pool is increased, it will be almost impossible for an electric system with the current structure to supply the total power required for the individualized charge, because a good part of the power produced by the system in order to face the losses, needs to be added to the power needed to charge the battery of each vehicle, increasing its inefficiency.

Due to its convenience, the individualized charge is the most desired option. What could be viable for the system of this type of charge would be the inclusion of renewable energies such as solar or photovoltaic. A house with renewable energy facilities will be able to charge a vehicle needing very little energy or even doing it isolated from the grid. This would allow the user with an electric vehicle to charge it at home without turning to any substation. Even with the integration of renewable energies, it will not be possible to perform quick charges because of the high power needed. Therefore, the presence of individualized charge "external" to the grid, does not exclude the presence of stations aimed to quick charge.

The most positive solution for the development of the electric vehicle lies in a deep change, at a development level, starting from the standardization of the batteries regarding size, capacities, location in the vehicle, etc. Besides, depending on the number of batteries in the station and the power demand, the charge current could be decreased and take advantage of the consumption valleys, which would make the charge even cheaper. If the procedure is performed, the vehicle could be charged slowly during the night in the house or in the private car park, stocking up from renewable sources and in case of needing a charge during the day, it would be possible to go to the nearest station to proceed to the change of battery. The solution suggested would allow long journeys regardless of the range of the vehicle; without the negative effects which quick or semi-quick charges have on the grid.

\section{References}

[1] Muhammad H. Rashid. "Electrónica de potencia: circuitos, dispositivos y aplicaciones" ( $3^{\mathrm{a}} \mathrm{ed}$.).

[2] Eva Molero Piñero "El vehículo eléctrico y su infraestructura de carga"

[3] Pascual Simon Comin "Cálculo y diseño de líneas eléctricas de alta tensión"

[4] B. Kramer, S. Chakraborty, and B. Kroposki, "A review of plug-in vehicles and vehicleto-grid capability," in Industrial Electronics, 2008. IECON 2008. 34th Annual Conference of IEEE, 2008, pp. 2278-2283.

[5] "Assessment of the Impact of Plug-in Electric Vehicles on Distribution Networks," IEEE Transactions on Power Systems, vol. 26, no. 1, pp. 206-213, Feb. 2011. 